\title{
Heavy Flavour Physics with ATLAS
}

\section{Ina Chalupkova on behalf of the ATLAS collaboration}

Charles University in Prague

Institute of Particle and Nuclear Physics

$16^{\text {th }}$ Lomonosov Conference on Elementary Particle Physics

27.8.2013
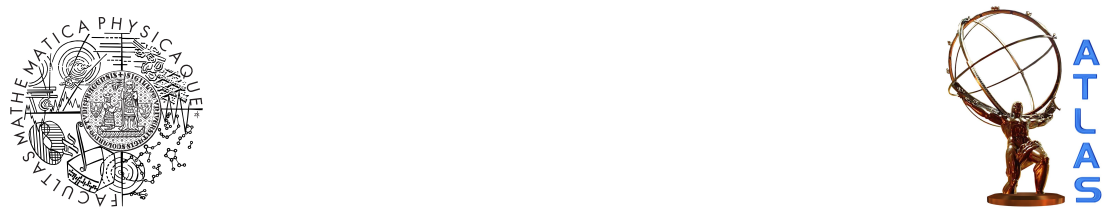

Ina Chalupkova (Charles University) 


\section{ATLAS detector}

Inner Detector: tracking, momentum and vertex measurement

- $|\eta|<2.5, \mathrm{~d}_{0}$ resolution $\sim 10 \mu \mathrm{m}$

Muon Spectrometer: trigger and muon identification

- $|\eta|<2.7$, resolution $\sim 40 \mu \mathrm{m}$

Mass resolution

- $\sigma\left(m_{\mathrm{J} / \psi}\right)=46 \pm 1 \mathrm{MeV}$
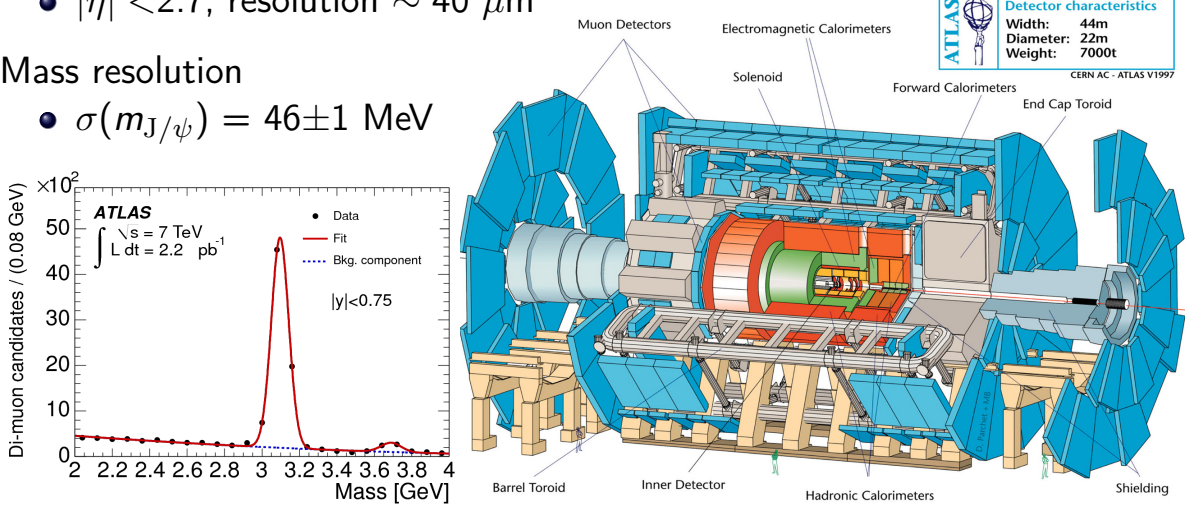
B-physics trigger:

- muon and di-muon decays $(\mathrm{J} / \psi, \Upsilon)$

- L1: single and di-muon triggers (thresholds 4-40GeV $p_{\mathrm{T}}$ )

- L2 and L3: muons from common vertex, opposite charge

- invariant mass window of $\mathrm{J} / \psi, \mathrm{B}$ and $\Upsilon$ un-prescaled

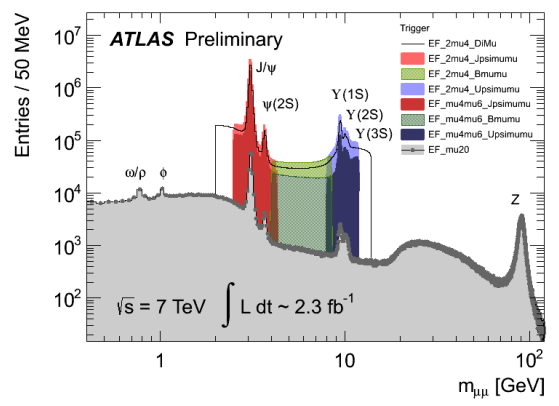

Status of analyses:

- focusing on searches for BSM physics in rare and semi-rare decays

- first observation of $\chi_{b}(3 \mathrm{P}), \mathrm{W}$ and prompt $\mathrm{J} / \psi$ production, $\mathrm{W}$ and charm production - M. Saleem

- all updated results on http://twiki.cern.ch/twiki/bin/view/AtlasPublic 


\section{$B^{+}$cross-section measurement in $\mathrm{B}^{+} \rightarrow \mathrm{J} / \psi \mathrm{K}^{+}$}




\section{$B^{+}$cross-section measurement in $\mathrm{B}^{+} \rightarrow \mathrm{J} / \psi \mathrm{K}^{+}$}

Analysis:

- find $\mathrm{J} / \psi$ candidates in $2.7-3.5 \mathrm{GeV}$

- fit to common vertex with another charged track

- select $\mathrm{B}^{+}$candidates with

$p_{\mathrm{T}}>9 \mathrm{GeV}$ and in $|\eta|<2.3$

- $2.4 \mathrm{fb}^{-1}$ data at $7 \mathrm{TeV}$

Background:

- resonant $\mathrm{J} / \psi \pi, \mathrm{J} / \psi \pi \mathrm{K}$

- combinatorial $\mathrm{J} / \psi \mathrm{X}$

Differential cross-section

$$
\begin{gathered}
\frac{\mathrm{d}^{2} \sigma\left(\mathrm{pp} \rightarrow \mathrm{B}^{+} \mathrm{X}\right)}{\mathrm{dp} \mathrm{p}_{\mathrm{T}}^{\mathrm{dy}}} \cdot \mathcal{B}=\frac{N^{\mathrm{B}^{+}}}{\mathcal{L} \cdot \Delta p_{\mathrm{T}} \cdot \Delta y} \\
N^{B^{+}}=\frac{1}{A} \frac{N_{\text {reco }}^{B^{+}}}{\varepsilon^{B^{+}}}=\frac{1}{A} \frac{N_{\text {reco }}^{B^{-}}}{\varepsilon^{B^{-}}}=\frac{1}{A} \frac{N_{\text {reco }}^{B^{ \pm}}}{\varepsilon^{B^{+}}+\varepsilon^{B^{-}}}
\end{gathered}
$$

arXiv:1307.0126 [hep-ex], sub. JHEP
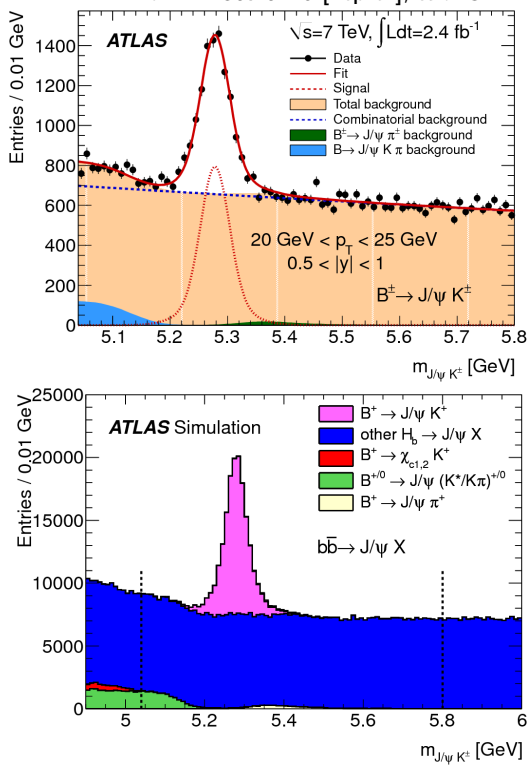


\section{$\mathrm{B}^{+}$cross-section measurement in $\mathrm{B}^{+} \rightarrow \mathrm{J} / \psi \mathrm{K}^{+}$}

Double differential cross-section compared with NLO MC
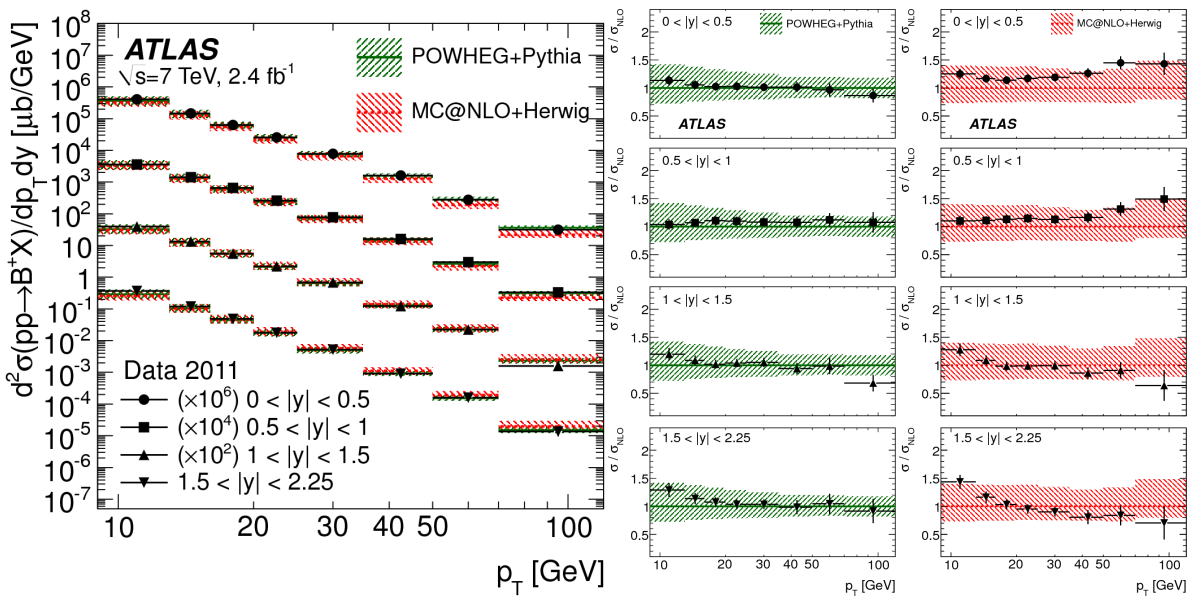

- good agreement with POWHEG + Pythia

- slightly higher cross-section at low $p_{\mathrm{T}}$ than MC@NLO + Herwig 


\section{$\mathrm{B}^{+}$cross-section measurement in $\mathrm{B}^{+} \rightarrow \mathrm{J} / \psi \mathrm{K}^{+}$}
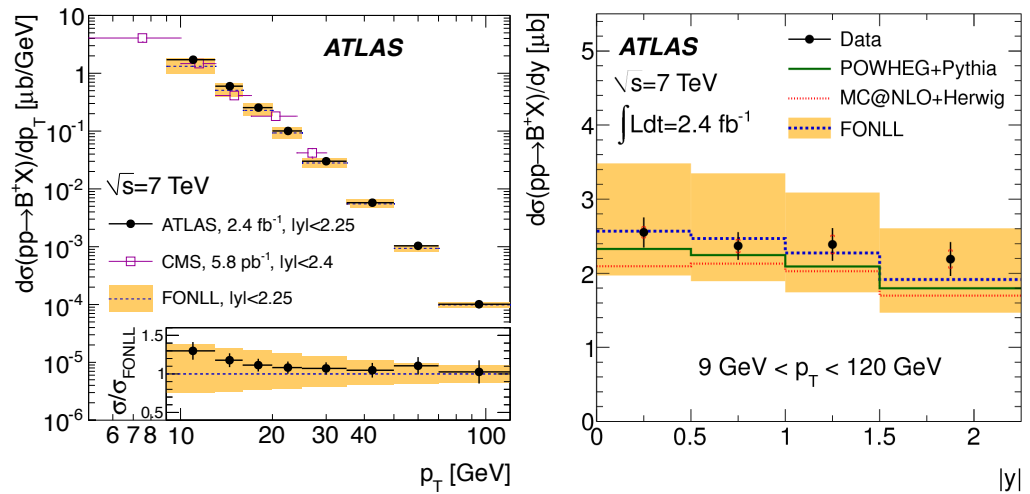

- compared to FONLL (Fixed-Order-Next-to-Leading-Logarithm)

- FONLL provides a good description for the data points

- integrated $B^{+}$production cross-section $\left(9 \mathrm{GeV}<p_{\mathrm{T}}<120 \mathrm{GeV}\right)$ $\sigma\left(p p \rightarrow B^{+} X\right)=10.6 \pm 0.3$ (stat.) \pm 0.7 (syst.) \pm 0.2 (lumi. $) \pm 0.4(\mathcal{B}) \mu \mathrm{b}$ 


\section{Angular analysis of $\mathrm{B}_{d}^{0} \rightarrow \mathrm{K}^{* 0} \mu^{+} \mu^{-}$}




\section{Angular analysis of $\mathrm{B}_{d}^{0} \rightarrow \mathrm{K}^{* 0} \mu^{+} \mu^{-}$}

ATLAS-CONF-2013-038 $b \rightarrow s$ transition allowed only at loop level in SM, search for new physics

Event selection: based on $\mathrm{K} \pi$ and $\mathrm{K} \pi \mu \mu$ invariant mass

- excluded resonant $\mathrm{J} / \psi$ and $\psi(2 \mathrm{~s})$

- MC-optimized cuts for combinatorial and radiative charmonium decays background

Analysis:

- use unbinned maximum likelihood fit

- fit mass to separate signal from background
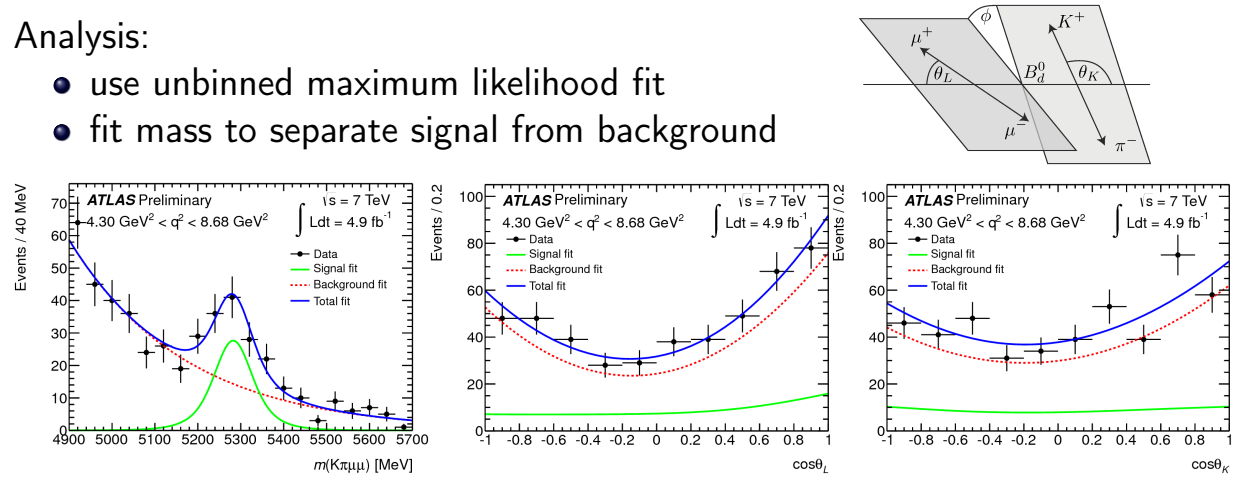


\section{Angular analysis of $\mathrm{B}_{d}^{0} \rightarrow \mathrm{K}^{* 0} \mu^{+} \mu^{-}$}

Forward-backward asymmetry and longitudinal polarization fraction

$\frac{1}{\Gamma} \frac{\mathrm{d}^{2} \Gamma}{\mathrm{d} q^{2} \mathrm{~d} \cos \theta_{L}}=\frac{3}{4} F_{L}\left(q^{2}\right)\left(1-\cos ^{2} \theta_{L}\right)+\frac{3}{8}\left(1-F_{L}\left(q^{2}\right)\right)\left(1+\cos ^{2} \theta_{L}\right)+$

$+A_{F B}\left(q^{2}\right) \cos \theta_{L}$
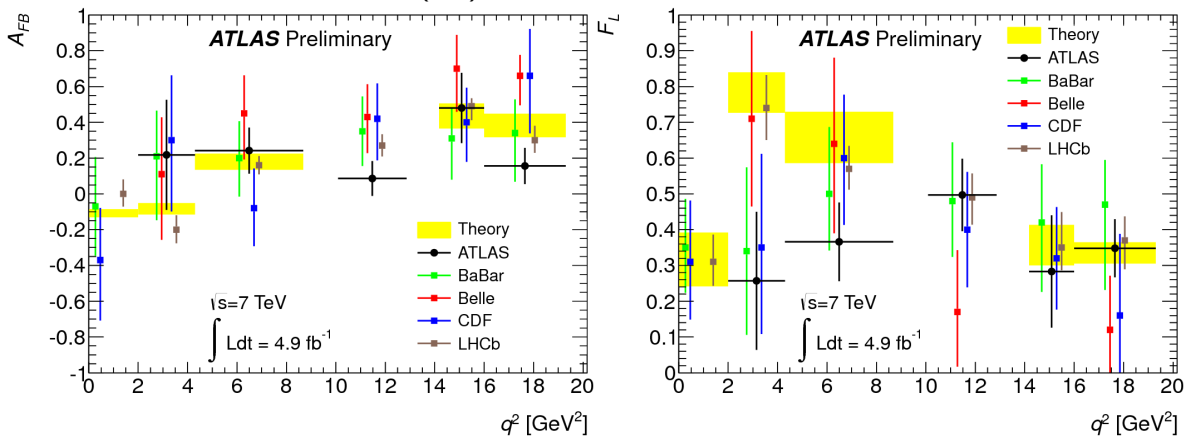

- measurement consistent with SM

- uncertainties statistically dominated (working on 2012 data) 


\section{$\mathrm{B}_{s}^{o} \rightarrow \mathrm{J} / \psi \phi$ : measurement of $\phi_{s}$ and $\Delta \Gamma_{s}$}




\section{$\mathrm{B}_{s}^{o} \rightarrow \mathrm{J} / \psi \phi$}

Standard Model predicts very small value for $B_{s}$ mixing phase

ATLAS-CONF-2013-039

$\phi_{s}=\arg \left(-\mathrm{M}_{12} / \Gamma_{12}\right) \sim-0.04 \pm 0.002 \sim-2 \beta_{s}$

Event selection: $\mathrm{J} / \psi$ dimuon trigger, reconstruction of $\mathrm{B}_{s} \rightarrow \mu^{+} \mu^{-} \mathrm{K}^{+} \mathrm{K}^{-}$ Measure mass and proper decay time $\tau=\frac{L_{x y} M_{B_{s}}}{p_{t_{B}}}$
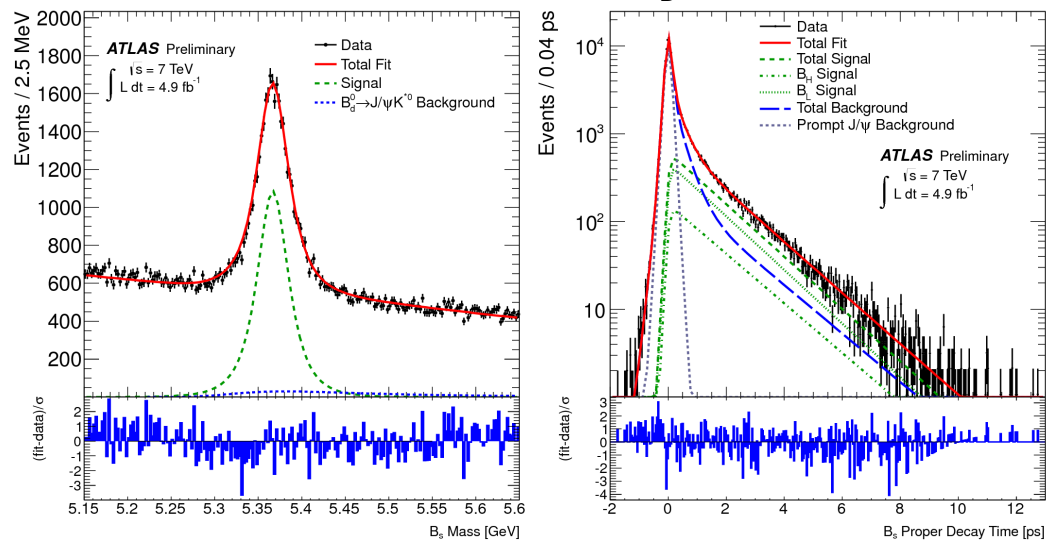


\section{$\mathrm{B}_{s}^{o} \rightarrow \mathrm{J} / \psi \phi$}

Flavour tagging to determine initial flavour eigenstate of $B_{s}$

- using opposite side tagging, studied with $B^{ \pm} \rightarrow J / \psi K^{ \pm}$

- muon tagger - muon from semileptonic decay, muon cone charge $Q_{\mu}$

- jet charge tagger - jet from the same primary vertex, jet charge $Q_{j e t}$

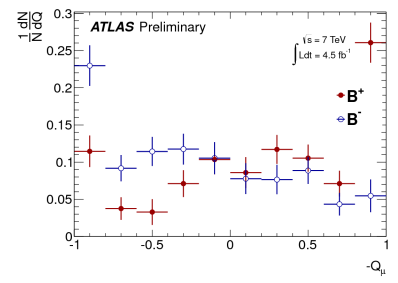

segment muons

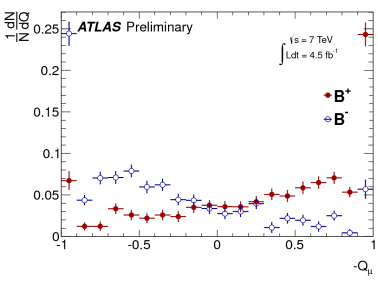

combined muons

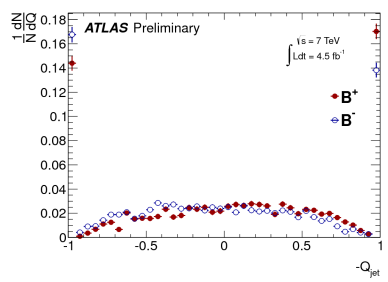

jet charge 


\section{$\mathrm{B}_{s}^{o} \rightarrow \mathrm{J} / \psi \phi$}

Transversity angles in signal region:
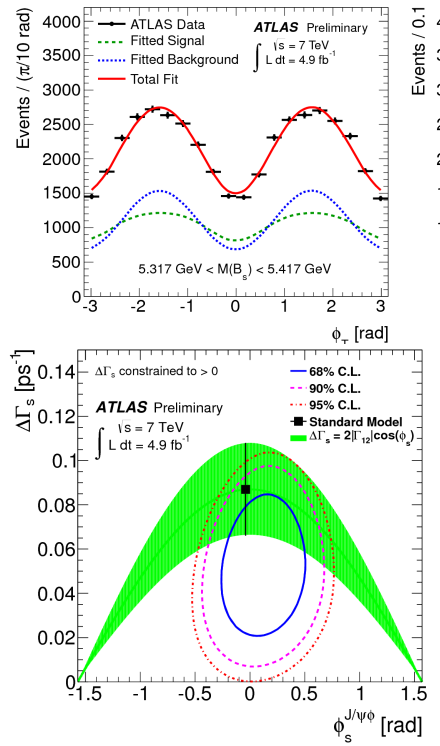
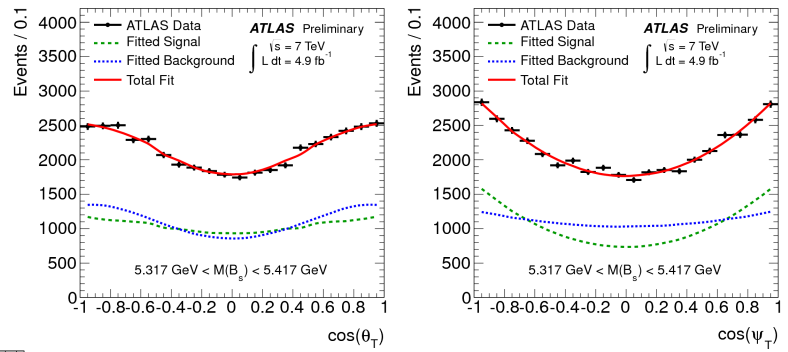

$$
\begin{aligned}
& \phi_{s}=0.12 \pm 0.25 \text { (stat.) } \pm 0.11 \text { (syst.) rad } \\
& \Delta \Gamma_{s}=0.053 \pm 0.021 \text { (stat.) } \pm 0.009 \text { (syst.) } \mathrm{ps}^{-1}
\end{aligned}
$$

- uncertainty of $\phi_{s}$ improved by $40 \%$ w.r.t. untagged analysis, dominated by statistical error 


\section{$\Lambda_{b} \rightarrow J / \psi \Lambda^{0}: \alpha_{b}$ and helicity}




\section{$\Lambda_{b} \rightarrow J / \psi\left(\mu^{+} \mu^{-}\right) \Lambda^{0}\left(\mathrm{p} \pi^{-}\right)$}

Selection:

- $\mathrm{J} / \psi: 2.8 \mathrm{GeV}<\mathrm{m}_{\mu \mu}<3.4 \mathrm{GeV}$

- $\Lambda^{0}: 1.08 \mathrm{GeV}<\mathrm{m}_{\mathrm{hh}}<1.15 \mathrm{GeV}$

- $\Lambda_{b}^{0}: 5.56 \mathrm{GeV}<\mathrm{m}_{\mathrm{J} / \psi^{\sim}}<5.68 \mathrm{GeV}$

- cascade topology: $\chi^{2} / N_{\text {dof }}<3$, $\mathrm{L}_{x y}>10 \mathrm{~mm}, \tau_{\Lambda_{b}}>0.35 \mathrm{ps}$

- $1400 \Lambda_{b}^{0}$ and $\bar{\Lambda}_{b}^{0}$

Analysis:

- decay described by 4 helicity amplitudes

- parity violating asymmetry parameter $\alpha_{b}=\left|a_{+}\right|^{2}-\left|a_{-}\right|^{2}+\left|b_{+}\right|^{2}-\left|b_{-}\right|^{2}$

- full angular PDF

- used method of moments $F_{i}$

- lifetime and mass measurement in Phys. Rev. D87 (2013) 032002

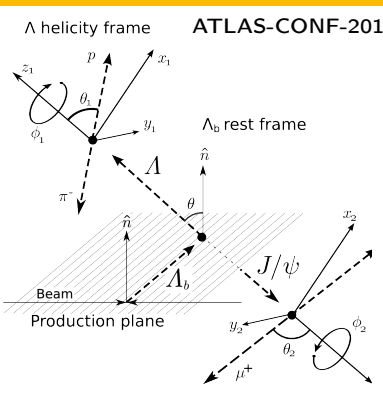

$\mathrm{J} / \Psi$ helicity frame

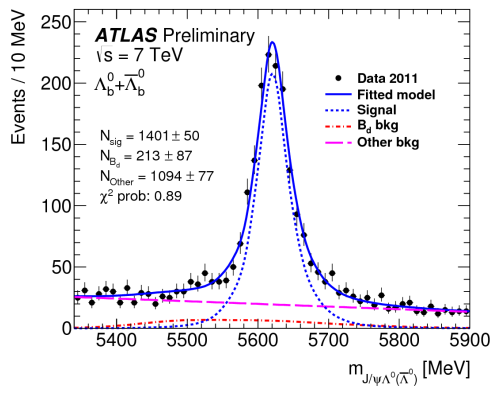




\section{$\Lambda_{b} \rightarrow J / \psi\left(\mu^{+} \mu^{-}\right) \Lambda^{0}\left(\mathrm{p} \pi^{-}\right)$}

Check fit: compared $F_{i}$ for data with weighted signal+background $\mathrm{MC}$

Results:

$$
\begin{aligned}
& \left|\mathrm{a}_{+}\right|=0.17_{-0.17}^{+0.12} \text { (stat) } \pm 0.06 \text { (syst) } \\
& \left|\mathrm{a}_{-}\right|=0.59_{-0.07}^{+0.06}(\text { stat }) \pm 0.04 \text { (syst) } \\
& \left|\mathrm{b}_{+}\right|=0.78_{-0.05}^{+0.04}(\text { stat }) \pm 0.02 \text { (syst) } \\
& \left|\mathrm{b}_{-}\right|=0.08_{-0.08}^{+0.13}(\text { stat }) \pm 0.05 \text { (syst) } \\
& \alpha_{b}=0.28 \pm 0.16 \text { (stat) } \pm 0.06 \text { (syst) }
\end{aligned}
$$

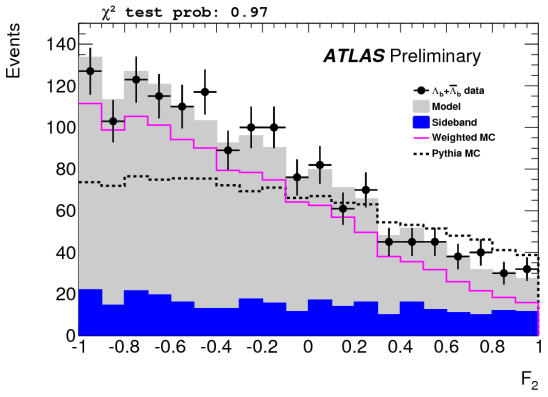

- $\wedge^{0}$ and $\mathrm{J} / \psi$ are highly polarized in direction of their momenta

- $\alpha_{b}$ value consistent with LHCb: $0.05 \pm 0.17$ (stat) \pm 0.07 (syst) (Phys. Lett.B724 (2013) 27) 


\section{Plans and upgrades}

Analysis plans:

- most of results are statistically limited

- more accurate measurements with 2012 data starting or ongoing ( $\chi_{c n}$ production and heavy flavour production nearly finished)

- $B_{s} \rightarrow \mu \mu$ : published analysis of 2011 data $\mathcal{B}\left(B_{s}^{0} \rightarrow \mu^{+} \mu^{-}\right)<1.5(1.2) \times 10^{-8}$ at $95 \%(90 \%) \mathrm{CL}$

Detector upgrades:

- IBL - 4th layer of Pixel detector $3.3 \mathrm{~cm}$ from IP

- TDAQ and trigger upgrades

- Fast track trigger $(2016 / 17)$

- new all-silicon ID (2022/23)

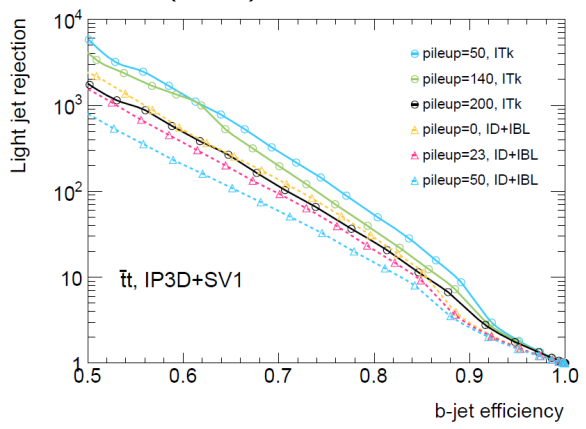




\section{Backup}




\section{$\mathrm{B}_{s}^{\circ} \rightarrow \mathrm{J} / \psi \phi:$ tagging}

\begin{tabular}{l|c|c|c}
\hline Tagger & Efficiency [\%] & Dilution [\%] & Tagging Power [\%] \\
\hline \hline Segment Tagged muon & $1.08 \pm 0.02$ & $36.7 \pm 0.7$ & $0.15 \pm 0.02$ \\
Combined muon & $3.37 \pm 0.04$ & $50.6 \pm 0.5$ & $0.86 \pm 0.04$ \\
Jet charge & $27.7 \pm 0.1$ & $12.68 \pm 0.06$ & $0.45 \pm 0.03$ \\
\hline Total & $32.1 \pm 0.1$ & $21.3 \pm 0.08$ & $1.45 \pm 0.05$ \\
\hline
\end{tabular}

Complete results:

$\phi_{s}=0.12 \pm 0.25$ (stat.) \pm 0.11 (syst.) $\mathrm{rad}$

$\Delta \Gamma_{s}=0.053 \pm 0.021$ (stat.) \pm 0.009 (syst.) $\mathrm{ps}^{-1}$

$\Gamma_{s}=0.677 \pm 0.007$ (stat.) \pm 0.003 (syst.) $\mathrm{ps}^{-1}$

$\left|A_{0}(0)\right|^{2}=0.529 \pm 0.006$ (stat.) \pm 0.011 (syst.)

$\left|A_{\|}(0)\right|^{2}=0.220 \pm 0.008$ (stat.) \pm 0.009 (syst.)

$\delta_{\perp}=3.89 \pm 0.46$ (stat.) \pm 0.13 (syst.) $\mathrm{rad}$

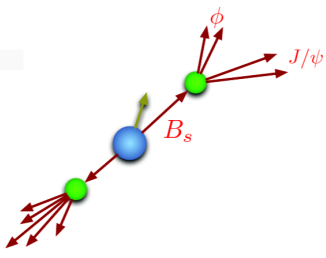




\section{Associated production of $\mathrm{W}$ and prompt $\mathrm{J} / \psi$}

Probes quarkonium production mechanism, sensitive to multiple parton interactions.

Selection:

- prompt $\mathrm{J} / \psi$ via mass and pseudo-proper time

- $\mathrm{W}^{ \pm}$muon trigger, identified by $\mu$ and missing $p_{\mathrm{T}}$

- fit $\mathrm{W}^{ \pm}$transverse mass with multijet background

- 29 events with $\mathrm{W}^{ \pm} \mathrm{J} / \psi$

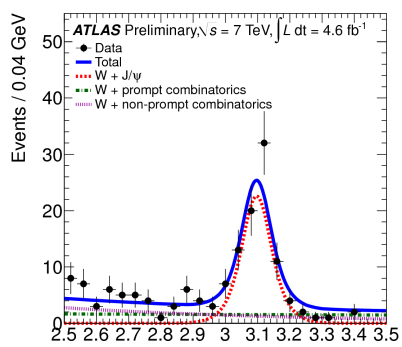

$\mu^{+} \mu^{-}$Invariant mass [GeV]

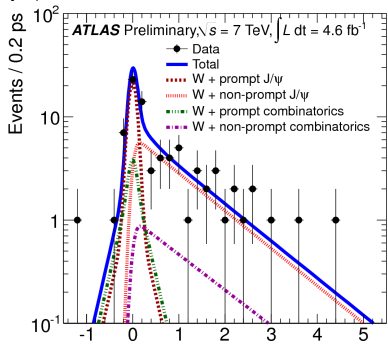

$\mathrm{J} / \psi$ Pseudo-proper time [ps]

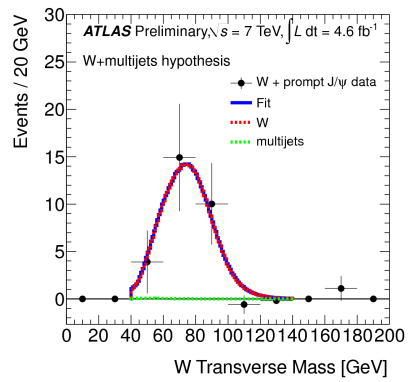

WTransverse Mass [GeV] 


\section{Associated production of $\mathrm{W}$ and prompt $\mathrm{J} / \psi$}

- double parton scattering estimate $\sim 40 \%$ from

$$
\mathrm{d} \sigma_{J / \psi \mid W}=\sigma_{W} \otimes \sigma_{J / \psi} / \sigma_{\text {eff }}
$$

- extract inclusive (SPS+DPS) cross-section ratio

- comparison with theory: measured rate underestimated (but large uncertainties of data)
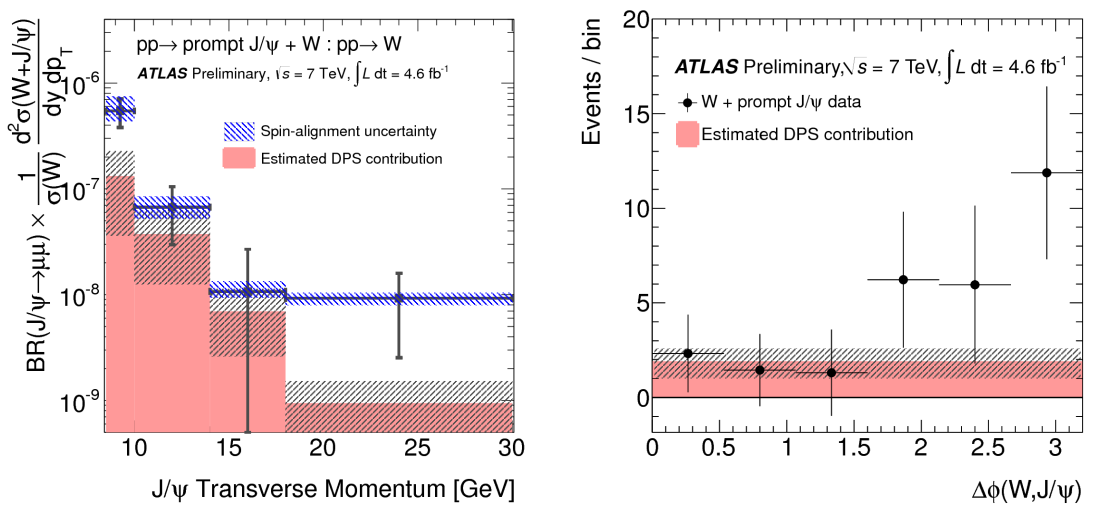